\title{
VARIACIÓN EN LA INTENSIDAD DE LA ISLA DE CALOR URBANA POR EFECTO DEL CAMBIO CLIMÁTICO EN CIUDADES CHILENAS
}

\author{
Cristian Henríquez \\ Instituto de Geografía, Pontificia Universidad Católica de Chile, \\ Centro de Desarrollo Urbano Sustentable (CEDEUS) \\ \& Centro Interdisciplinario de Cambio Global UC \\ cghenriq@uc.cl \\ Pamela Smith \\ Departamento de Geografía, Universidad de Chile, \\ Centro de Ciencia del Clima y la Resiliencia (CR) \\ pamelasmit@uchilefau.cl \\ Paulina Contreras \\ plcontreras@uc.cl \\ Jorge Qüense \\ jquense@uc.cl
}

Instituto de Geografía, Pontificia Universidad Católica de Chile

\begin{abstract}
Resumen: La Isla de Calor Urbano (ICU) es la principal característica del clima urbano y se define como la diferencia entre la temperatura urbana y la temperatura rural. Su existencia se explica por el diseño y materiales de construcción de la ciudad, superficies impermeables y no evapotranspirantes, y sus efectos son de diversa índole. La magnitud e intensidad de la ICU depende, a su vez, del tamaño de la ciudad y por ello, del volumen de población concentrada espacialmente. El objetivo de esta investigación es proponer una metodología que permita estimar la intensidad máxima de la ICU presente y futura mediante una aproximación de múltiples modelos (climáticos y de usos de suelo) y considerando los efectos del cambio climático. Los resultados demuestran que la ICU prome-
\end{abstract}


dio de las principales ciudades chilenas analizadas es consistente con valores de estudios internacionales, destacando el caso del Gran Santiago que llegaría a tener una ICU mayor $10{ }^{\circ} \mathrm{C}$.

Palabras clave: Isla de calor urbana, Modelo de cambio de uso de suelo, Cambio climático, Estimación de la ICU, ARClim.

\title{
Variation in the intensity of the urban heat island due to climate change in Chilean cities
}

\begin{abstract}
Urban Heat Island (UHI) is the main characteristic of the urban climate and is defined as the difference between urban and rural temperature. Its existence is explained by the design and construction materials of the city, impermeable and non-evapo-transpiring surfaces, its effects are of various kinds. The magnitude and intensity of the ICU depends on the size of the city and therefore, the volume of spatially concentrated population. The objective of this research is to propose a methodology that allows estimating the maximum intensity present and future ICU through an approximation of multiple models (climate and land use) and considering the effects of climate change. The results show that the average ICU of the main Chilean cities analyzed is consistent with values from international studies, highlighting the case of Great Santiago, which would have a UCI higher than $10{ }^{\circ} \mathrm{C}$.
\end{abstract}

Keywords: Urban heat island, Land use change model, Climate change, UHI estimation, ARClim.

\section{Introducción}

El cambio climático plantea distintas amenazas meteorológicas que pueden afectar significativamente a las ciudades, especialmente a las edificaciones y los espacios públicos que juegan un rol clave en la naturaleza propia de la ciudad y del habitar urbano. El clima urbano, junto con el impacto del cambio climático, tendrán importantes efectos sobre la intensidad de la isla de calor urbana y, a su vez, sobre la salud y bienestar de la población, en especial sobre el disconfort térmico y la demanda energética para climatización, entre otros efectos (Henríquez et al., 2019). En los últimos años se ha observado una intensificación de los extremos climáticos, tales como el aumento de olas de calor y temperaturas máximas que afectan a las ciudades en el período estival. Este aumento de la temperatura provoca condiciones de disconfort térmico tanto al interior de las edificaciones, como en los espacios exteriores, como plazas, parques y espacios públicos en general, afectando especialmente a la población más vulnerable.

El aumento de la temperatura por el cambio climático puede intensificar el efecto de Isla de Calor Urbana (ICU). Este fenómeno corresponde a la principal característica 
del clima urbano y se define como la diferencia entre la temperatura urbana y la temperatura rural (Oke, 1987). Su existencia se explica por el diseño y materiales de construcción de la ciudad, superficies impermeables y no evapotranspirantes, tales como, asfalto y concreto, que generalmente tienen una alta absorción de la radiación solar y una alta capacidad termal y de conductividad, además de una menor cobertura vegetal, que disminuye la humedad y aumenta el calor (Ochoa de la Torre et al, 2009; Smith \& Romero, 2016). La magnitud e intensidad de la ICU, depende a su vez, del tamaño de la ciudad y por ello, del volumen de población concentrada espacialmente.

Las consecuencias asociadas a la ICU son de diversa índole, como florecimiento temprano de la vegetación urbana, formación de ozono troposférico, aumento de días con polución atmosférica y alteración del confort bioclimático. Además, las quemas agrícolas que circundan la ciudad producen humo y material particulado en suspensión que son atraídos por la presencia de la ICU. También están las emanaciones de monóxido de carbono (CO) e hidrocarburos del parque automotor, especialmente de la locomoción colectiva. Del mismo modo, la emisión de gases contaminantes de las industrias intra y extraurbanas, junto con la existencia de calles sin pavimentar y la congestión vehicular en horas puntas, contribuyen a la contaminación del aire, especialmente en áreas donde la intensidad de la ICU es significativa (Henríquez, 2014).

La ICU tiene una influencia importante en la eficiencia energética de edificios y en el disconfort residencial asociado al calor, especialmente nocturno. Para combatir este fenómeno se usan sistemas de enfriamiento del aire (aire acondicionado), sin embargo, la mayor parte de las viviendas latinoamericanas no disponen de estos sistemas, especialmente en el caso de viviendas precarias y barrios marginales, como son los campamentos en Chile. Los efectos indirectos de esta se sienten fundamentalmente en la población infantil y anciana, a través de una mayor sensación de disconfort térmico, disminución de la calidad de vida e incluso enfermedades respiratorias en los meses estivales cuando se producen los episodios de ICU más intensos.

La ICU se produce con mayor intensidad en el centro urbano donde hay mayor densidad y altura de edificios (Figura 1), donde el calor se concentra y luego se libera lentamente en las horas siguientes a la puesta de sol. Sin embargo, el efecto se extiende por toda la superficie urbana, especialmente en ciudades de gran tamaño y de tamaño medio.

Romero, Ordenes, \& Vásquez (2003) han reportado la existencia de varias islas de calor urbanas en Santiago y otras ciudades medias chilenas, configurando una estructura de archipiélagos de calor urbanos (ACU), los cuales facilitan la convergencia de plumas de contaminación hacia los sectores más cálidos, normalmente el centro de la ciudad u otros sectores con alta concentración de actividades. Dichos estudios, basados en el análisis de imágenes satelitales, señalan con nitidez la relevancia del número, magnitud y forma de los archipiélagos de calor urbanos en la configuración térmica de la ciudad, los que dependen de varios factores, tales como el uso de suelo, diseño de calles y edificios, altura de edificios, los materiales superficiales y, especialmente, de la distribución de parques (islas de frío urbanas). 


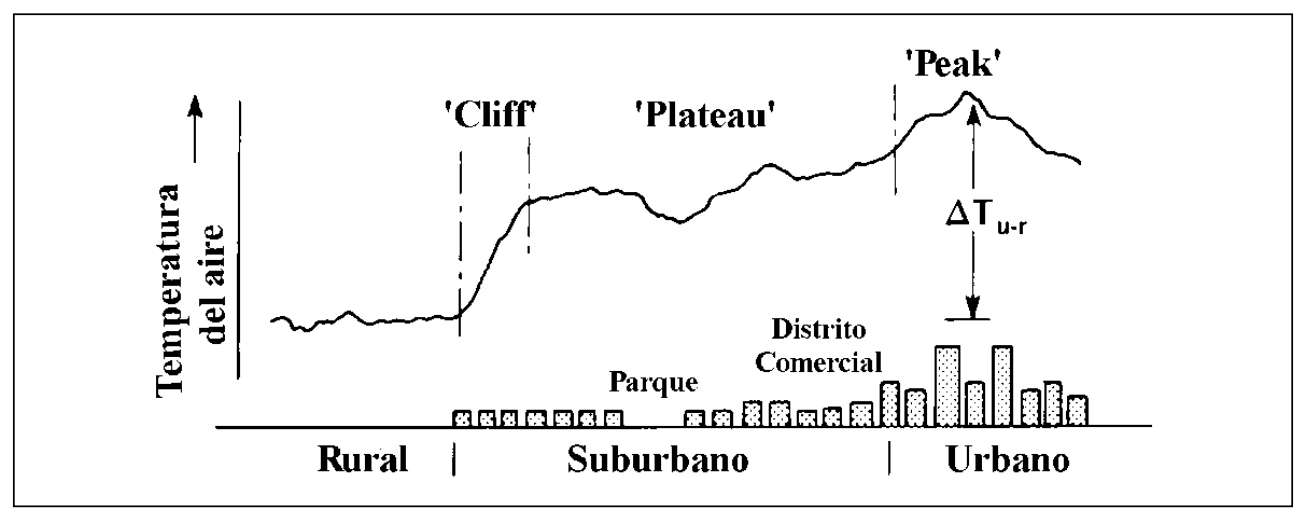

Figura 1. Transecto tipo de una isla de calor urbana.

Fuente: Modificado de Oke (1976) en Oke (1987:288).

Del mismo modo, se han observado efectos positivos de la isla de calor urbana y archipiélago de calor urbano en invierno en términos de confort bioclimático, especialmente los días de más bajas temperaturas. Sin embargo, en verano durante los días más calurosos sus efectos son claramente deletéreos (Whitford et al, 2001). Este impacto se centra en los meses de mayor calor y se espera que aumente con el cambio climático, considerando el aumento de las temperaturas máximas diarias. Las ICU en sí mismas no son producto del cambio climático, pero sin duda pueden verse aumentadas considerablemente por este aunque no necesariamente en su intensidad de manera directa.

En este contexto el propósito del presente artículo es evaluar la amenaza presente y futura de la intensidad máxima de la ICU en las principales ciudades chilenas, considerando el efecto del cambio climático. Esta propuesta metodológica es un aporte novedoso para la climatología urbana, cuyos resultados pueden ser de gran utilidad para la planificación y gestión ambiental.

\section{Materiales y Métodos}

\section{1. Área de Estudio}

La definición de las ciudades para esta investigación se ha basado en el criterio de tamaño de población, seleccionando ciudades o conurbaciones mayores a 50.000 habitantes y las capitales regionales del país, de acuerdo a la clasificación de población del documento Ciudades, Pueblos y Aldeas del Instituto Nacional de Estadística (INE, 2019). Lo anterior fue complementado con el criterio de Área Urbana Consolidada (AUC), término desarrollado por el Instituto Nacional de Estadística (INE) y el Ministe- 
rio de Vivienda y Urbanismo (MINVU) (INE \& MINVU, 2018), de manera de identificar las áreas urbanas conurbadas, que conforman una única unidad urbana morfológica. De esta forma, se han considerado 34 ciudades, incluyendo las ciudades de Coyhaique, región de Aysén, y la localidad de Hanga Roa en Isla de Pascua, región de Valparaíso, que tienen una población bajo este umbral, pero que permiten representar la amplia variedad climática del país (Figura 2).

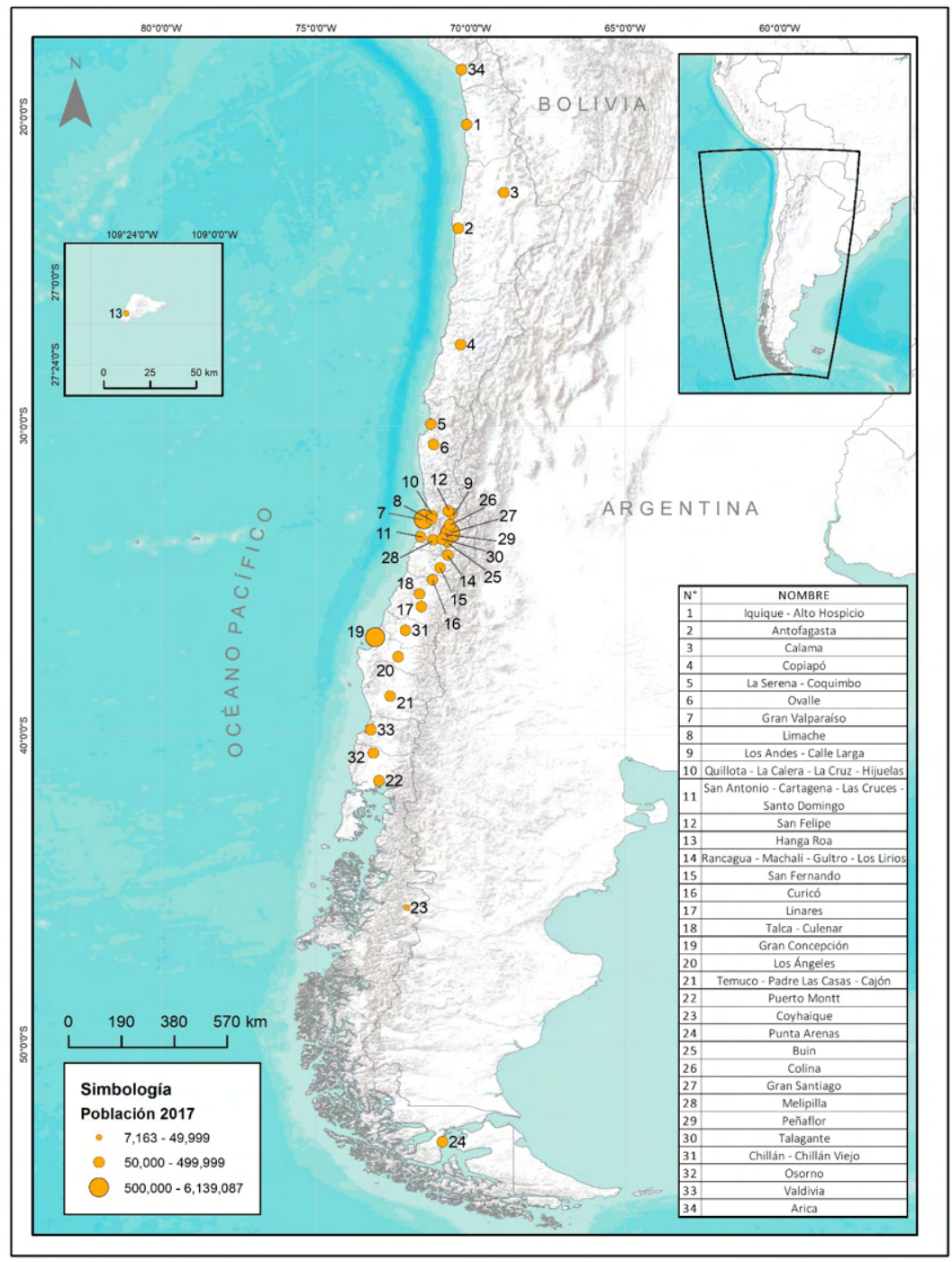

Figura 2. Mapa de área de estudio: ciudades chilenas seleccionadas.

Fuente: Elaboración propia. 


\section{Metodología}

Existen distintos métodos para evaluar la intensidad de la ICU: mediciones usando estaciones meteorológicas entre la ciudad y el campo adyacente a esta, con datos de temperatura superficial mediante imágenes satelitales, con dispositivos registradores portátiles o dataloggers de temperatura que se pueden colocar en distintos usos de suelo o con transectos móviles que registren la temperatura entre el centro de la ciudad y el área rural circundante. Todas estas aproximaciones se pueden complementar, ya que cada una tiene ventajas y desventajas, por ejemplo, integrando datos de estaciones meteorológicas en varios puntos de la ciudad, pero esta última información pocas veces está disponible en grandes ciudades -normalmente se encuentran en los aeropuertos- y es prácticamente inexistente en ciudades medias o pequeñas. Por esta razón se recurrió a métodos indirectos; primero, usando un modelo que relaciona la intensidad máxima de la ICU con la cantidad de población urbana y la velocidad del viento mediante la fórmula de Oke (1987), y el segundo, a datos meteorológicos presentes (1980 y 2010) y futuros (2035-2065) bajo el bajo el escenario RCP8.5. Las variables climáticas fueron sometidas a un proceso de escalamiento (downscaling) llevándolas a una retícula de cobertura nacional con resolución de $5 \times 5 \mathrm{~km}^{2}$. Los datos fueron generados por Meteodata (https://www.meteodata.cl/) y desarrollados en el contexto del proyecto Atlas de Riesgos Climáticos (ARClim) bajo la coordinación del Ministerio de Medio Ambiente en Chile. ARClim (https://arclim.mma.gob.cl/) es un proyecto del Ministerio del Medio Ambiente del Gobierno de Chile, desarrollado por el Centro de Investigación del Clima y la Resiliencia (CR2) y el Centro de Cambio Global (CCG-Universidad Católica de Chile) con la colaboración de otras instituciones nacionales y el financiamiento internacional del Ministerio Federal de Cooperación Económica y Desarrollo (BMZ) de Alemania.

En relación con la primera parte del modelo, el método de Oke supone que el tamaño de la ciudad puede ser sustituido por la relación logarítmica de la población (P). Para una situación "ideal" de vientos tranquilos y cielos despejados, la máxima ICU estaría muy correlacionado con el $\log \mathrm{P}$, según fue demostrado para varias localidades norteamericanas y europeas. Oke ha observado que incluso pequeños pueblos (población de 1.000 habitantes) tienen una isla de calor, donde los centros comerciales y pequeños grupos de edificios son más cálidos que sus alrededores. Por el otro extremo, se ha observado que la máxima ICU puede alcanzar los $12^{\circ} \mathrm{C}$ de diferencia entre la temperatura del centro de la ciudad y el entorno rural.

De esta forma se ha optado por usar este método debido a que no hay registros empíricos ni estimaciones comparables para todas las ciudades y además permite estimar la proyección futura. La ecuación original formulada por Oke para la estimación de la intensidad presente de la ICU es la siguiente: 


$$
\Delta I C U_{u-r}=\frac{P^{0,27}}{4 *(\underline{u})^{0,56}} \quad(\text { Ecuación 1) }
$$

Donde,

- $\Delta I C U_{u-r}$ es la diferencia máxima de temperatura entre el campo y ciudad en grados Celsius, correspondiente a la ICU presente

- $P$ es la población urbana presente,

- $\underline{u}$ es la velocidad del viento presente en m/s a una altura de 10 metros en un área rural próxima a la ciudad

Esta fórmula fue ideada para el atardecer con cielos despejados. Obviamente con fuertes vientos urbano/rurales la diferencia termal disminuye (adaptada de Oke, 1987).

La adaptación de la ecuación de Oke de estimación de intensidad máxima de ICU considerando el efecto de cambio climático es la siguiente:

$$
\Delta I C U C C_{u-r}=\frac{P^{0,27}}{4 *(\underline{u})^{0,56}}+\Delta \operatorname{Tmin} \quad \text { (Ecuación 2) }
$$

Donde,

- $\triangle I C U C C_{u-r}$ es la diferencia máxima de temperatura entre el campo y ciudad en grados Celsius, correspondiente a la ICU futura incluyendo el efecto de cambio climático

- $P$ es la población urbana futura,

- $\underline{u}$ es la velocidad del viento futura en $\mathrm{m} / \mathrm{s}$ a una altura de 10 metros en un área rural próxima a la ciudad

- $\Delta$ Tmin es la diferencia de temperatura mínima diaria promedio futura de verano menos la temperatura mínima diaria promedio presente en grados Celsius, atribuida al efecto del cambio climático

La población urbana presente se obtiene de los registros censales, en este caso del Censo de Población y Vivienda realizado por el Instituto Nacional de Estadística (INE) del año 2017. Para el caso de la población urbana futura se usó un modelo de cambio de uso de suelo que permite estimar la superficie urbana proyectada al 2065, y con ello la cantidad de población mediante un modelo de regresión lineal simple. Considerando las rápidas dinámicas urbanas, se ha desarrollado un modelo de cambio de uso de suelo con el mismo horizonte temporal de los modelos climáticos de ARClim para determinar la exposición futura a través de las superficies urbanas simuladas y para estimar la 
ICU a través de la población inferida del modelo. Para la obtención del uso urbano del año 2065 se utilizó un modelo de predicción de cambio de uso de suelo, mediante el módulo de IDRISI, Land Change Modeler, para las ciudades principales del país. Este modelo consideró seis fuerzas motrices correspondientes a pendiente del terreno, distancia a red vial estructurante, distancia a ciudades principales, distancia a ciudades secundarias, usos de suelo y densidad poblacional. Como base se utilizaron las coberturas de mancha urbana provistas por el Ministerio de Vivienda y Urbanismo (MINVU) para los años 1992, 2002 y 2017 y como restricciones se usaron las Áreas Silvestres Protegidas del Estado (SNASPE). Luego se realizó una regresión logística entre el uso urbano y las fuerzas motrices. Para estimar las demandas de uso urbano se aplicó el método de cadenas de Markov. Todo esto permitió modelar el uso urbano al año 2065. Para validar el modelo se proyectó al año 2017 y se comparó con el año 2017 observado mediante el índice Kappa. Finalmente, mediante una regresión lineal entre los datos de población de "Ciudades, pueblos, aldeas y caseríos" de los censos de 1992 y 2017 y la superficie real del MINVU 1992, 2002 y 2017 y la obtenida mediante la modelación al año 2065, se proyectó la población urbana al año 2065.

Por su parte, la velocidad del viento (viento máximo diario) se obtuvo de los registros meteorológicos para los escenarios presentes y futuros de los datos ARClim para el promedio de los meses de diciembre, enero y febrero (verano). Para determinar el punto rural próximo a la ciudad que requiere la fórmula de Oke se consideraron los siguientes criterios: que se encuentre en la misma zona climática de Köppen de la ciudad (Sarricolea et al, 2017), que la distancia a los cursos y cuerpos de agua, incluyendo la costa, sea equivalente a la del punto urbano (ciudades costeras), que no se encuentre sobre humedales; que no tenga una diferencia mayor a 200 metros en altitud del punto urbano; que sea representativo del contexto rural que rodea a la ciudad y que se mantenga en área rural según escenario de modelación 2065. Para las ciudades conurbadas se consideró un único punto rural.

Para estimar el efecto del cambio climático a la intensidad futura de la ICU al año 2065, se sumó la diferencia de temperatura mínima diaria promedio ( $\left.{ }^{\circ} \mathrm{C}\right)$ entre el escenario futuro y presente de los meses de diciembre, enero y febrero (DEF) para cada ciudad, a partir de los datos de ARClim. Al igual que en el caso del viento, la celda utilizada para el cálculo de temperaturas mínimas corresponde al punto rural próximo a la ciudad, tal como se explica en el párrafo anterior.

Para validar los resultados de la intensidad de la ICU estimada a partir de la adaptación de la fórmula de Oke, se compararon con valores de intensidad de la ICU nocturna calculada a partir de distintas aproximaciones.

- En primer lugar, se utilizaron los datos de temperaturas mínimas para los puntos rurales y urbanos representativos de cada ciudad generados por Meteodata para evaluar la ICU nocturna de verano (DEF) en el periodo presente. En este caso, 
Tabla 1. Población y viento presente y futuro

\begin{tabular}{|c|c|c|c|c|c|}
\hline $\begin{array}{l}\text { ID } \\
\text { mapa }\end{array}$ & Ciudades & $\begin{array}{c}\text { Población } \\
2017\end{array}$ & $\begin{array}{c}\text { Población } \\
2065\end{array}$ & $\begin{array}{l}\text { Velocidad del } \\
\text { viento presente } \\
\qquad(\mathrm{m} / \mathrm{s})\end{array}$ & $\begin{array}{c}\text { Velocidad del } \\
\text { viento futuro } \\
(\mathrm{m} / \mathrm{s})\end{array}$ \\
\hline 34 & Arica & 202131 & 231549 & 3,37 & 3,38 \\
\hline 1 & Iquique & 293068 & 357561 & 4,72 & 4,72 \\
\hline 2 & Antofagasta & 348517 & 460882 & 3,94 & 3,95 \\
\hline 3 & Calama & 157575 & 255683 & 5,60 & 5,61 \\
\hline 4 & Copiapó & 150804 & 196788 & 4,94 & 4,95 \\
\hline 5 & La Serena & 399450 & 657577 & 3,76 & 3,76 \\
\hline 6 & Ovalle & 75864 & 100612 & 3,59 & 3,62 \\
\hline 12 & San Felipe & 64543 & 207266 & 2,48 & 2,54 \\
\hline 9 & Los Andes & 69041 & 149416 & 2,48 & 2,54 \\
\hline 10 & Quillota & 149159 & 260235 & 2,62 & 2,63 \\
\hline 8 & Limache & 50998 & 128182 & 3,49 & 3,47 \\
\hline 7 & Gran Valparaíso & 896528 & 982832 & 8,15 & 7,92 \\
\hline 11 & San Antonio & 118668 & 155391 & 4,44 & 4,33 \\
\hline 13 & Hanga Roa & 7163 & 15608 & 13,32 & 12,82 \\
\hline 26 & Colina & 88858 & 266399 & 3,03 & 3,05 \\
\hline 27 & Gran Santiago & 6139087 & 6903681 & 2,95 & 2,97 \\
\hline 29 & Peñaflor & 82959 & 93700 & 3,05 & 3,06 \\
\hline 28 & Melipilla & 72212 & 187157 & 3,48 & 3,47 \\
\hline 30 & Talagante & 56878 & 111069 & 3,75 & 3,77 \\
\hline 25 & Buin & 65607 & 100163 & 4,22 & 4,22 \\
\hline 14 & Rancagua & 290029 & 512490 & 3,56 & 3,58 \\
\hline 15 & San Fernando & 64543 & 113788 & 4,15 & 4,19 \\
\hline 16 & Curicó & 125275 & 214167 & 5,08 & 5,12 \\
\hline 17 & Linares & 73602 & 134453 & 5,44 & 5,39 \\
\hline 18 & Talca & 236347 & 386175 & 5,22 & 5,22 \\
\hline 31 & Chillán & 164270 & 196135 & 6,69 & 6,59 \\
\hline 19 & Gran Concepción & 719944 & 801687 & 4,52 & 4,48 \\
\hline 20 & Los Ángeles & 143023 & 160066 & 7,42 & 7,32 \\
\hline 21 & Temuco & 277529 & 318179 & 4,57 & 4,36 \\
\hline 32 & Osorno & 147666 & 164494 & 5,92 & 5,85 \\
\hline 33 & Valdivia & 150048 & 224555 & 5,30 & 5,17 \\
\hline 22 & Puerto Montt & 169736 & 218104 & 5,28 & 5,10 \\
\hline 23 & Coyhaique & 49667 & 95751 & 7,06 & 7,10 \\
\hline \multirow[t]{2}{*}{24} & Punta Arenas & 123403 & 135661 & 8,23 & 8,29 \\
\hline & Total/promedio & 12224192 & 15497454 & 4,88 & 4,84 \\
\hline
\end{tabular}

Fuente: Elaboración propia y Meteodata. 
el punto urbano seleccionado corresponde a la zona urbana más cálida de acuerdo con la temperatura de emisión superficial nocturna promedio de verano, calculada sobre imágenes satelitales MODIS nocturna (0:00 h), entre los años 2008 y 2018. Mientras que el punto rural de referencia corresponde al mismo utilizado para calcular el viento rural.

- Considerando la información satelital de dichos puntos, se obtuvo también la Isla de Calor Urbana de Superficie (ICUs) a partir de la diferencia entre la temperatura de emisión superficial nocturna promedio de verano urbana y rural de verano entre los años 2008 y 2018, calculada sobre imágenes satelitales MODIS nocturna $(0: 00 \mathrm{~h})$.

- Luego, se seleccionaron 10 ciudades ubicadas en distintas latitudes, costeras e interiores, a modo de ejemplo, en función de los datos disponibles. En estas se calculó la ICU nocturna de verano calculada a partir de datos instrumentales registrados por estaciones meteorológicas fijas de redes de monitoreo oficiales (Dirección Meteorológica de Chile, SINCA y Agromet) instaladas en el interior y el área rural de cada ciudad.

- Finalmente se calculó la ICU nocturna de verano calculada a partir de registros de temperatura del aire obtenidas con dataloggers (HOBO) instalados en distintos usos de suelo urbanos y rurales. Estos registradores miden la temperatura del aire cada una hora y son parte de los Proyectos Fondecyt 1100657 y 1130305.

\section{Resultados}

Tanto la población urbana presente y futura como la velocidad del viento presente y futura se muestra en la siguiente tabla. Estos son los dos insumos básicos para aplicar la fórmula de Oke para la ICU presente y futura. Se observa que la población urbana total aumentaría sobre tres millones de personas al 2065, mientras que el viento no sufriría mayor variación.

La intensidad de la ICU promedio del conjunto de ciudades chilenas estudiadas mediante el método de Oke para el período presente es de $2,86^{\circ} \mathrm{C}$. La ciudad de Santiago presenta la mayor ICU con un valor estimado de $9,2^{\circ} \mathrm{C}$, mientras que Hanga Roa registra el menor valor con $0,6^{\circ} \mathrm{C}$, donde prácticamente no se evidencia el fenómeno. Para el futuro (2065) se estima, usando la adaptación a la ecuación de Oke, que el valor promedio de la ICU será de $4,26^{\circ} \mathrm{C}$. Este cálculo considera la población 2065 estimada a partir de la superficie simulada con el modelo IDRISI, el viento proyectado por Meteodata y el aporte de la diferencia entre las temperaturas mínimas promedio de verano presente y futuro, como incremento asociado al cambio climático (Figura 2). De esta forma se puede estimar un aumento de la intensidad en la ICU de $1,4^{\circ} \mathrm{C}$ (Tabla 2). 
Tabla 2. Isla de Calor Urbana presente, futura y diferencia

\begin{tabular}{|c|c|c|c|c|}
\hline ID mapa & Ciudades & ICU presente $\left({ }^{\circ} \mathrm{C}\right)$ & ICU futuro $\left({ }^{\circ} \mathrm{C}\right)$ & Delta $\left({ }^{\circ} \mathrm{C}\right)$ \\
\hline 34 & Arica & 3,43 & 5,11 & 1,68 \\
\hline 1 & Iquique & 3,14 & 4,75 & 1,61 \\
\hline 2 & Antofagasta & 3,64 & 5,30 & 1,66 \\
\hline 3 & Calama & 2,41 & 4,03 & 1,62 \\
\hline 4 & Copiapó & 2,56 & 3,84 & 1,28 \\
\hline 5 & La Serena & 3,88 & 5,32 & 1,44 \\
\hline 6 & Ovalle & 2,54 & 3,68 & 1,14 \\
\hline 12 & San Felipe & 2,99 & 5,02 & 2,03 \\
\hline 9 & Los Andes & 3,04 & 4,68 & 1,63 \\
\hline 10 & Quillota & 3,64 & 5,09 & 1,45 \\
\hline 8 & Limache & 2,32 & 3,79 & 1,47 \\
\hline 7 & Gran Valparaíso & 3,12 & 3,76 & 0,64 \\
\hline 11 & San Antonio & 2,54 & 3,54 & 0,99 \\
\hline 13 & Hanga Roa & 0,64 & 2,59 & 1,95 \\
\hline 26 & Colina & 2,92 & 4,86 & 1,94 \\
\hline 27 & Gran Santiago & 9,27 & 10,48 & 1,21 \\
\hline 29 & Peñaflor & 2,85 & 3,84 & 0,99 \\
\hline 28 & Melipilla & 2,55 & 4,11 & 1,57 \\
\hline 30 & Talagante & 2,29 & 3,59 & 1,29 \\
\hline 25 & Buin & 2,23 & 3,32 & 1,09 \\
\hline 14 & Rancagua & 3,66 & 5,23 & 1,56 \\
\hline 15 & San Fernando & 2,24 & 3,58 & 1,34 \\
\hline 16 & Curicó & 2,39 & 3,64 & 1,24 \\
\hline 17 & Linares & 2,00 & 3,43 & 1,44 \\
\hline 18 & Talca & 2,80 & 4,20 & 1,41 \\
\hline 31 & Chillán & 2,21 & 3,49 & 1,29 \\
\hline 19 & Gran Concepción & 4,10 & 5,33 & 1,23 \\
\hline 20 & Los Ángeles & 2,01 & 3,31 & 1,30 \\
\hline 21 & Temuco & 3,15 & 4,70 & 1,55 \\
\hline 32 & Osorno & 2,30 & 3,76 & 1,46 \\
\hline 33 & Valdivia & 2,45 & 3,95 & 1,50 \\
\hline 22 & Puerto Montt & 2,54 & 3,66 & 1,12 \\
\hline 23 & Coyhaique & 1,55 & 2,90 & 1,35 \\
\hline 24 & Punta Arenas & 1,81 & 2,86 & 1,04 \\
\hline
\end{tabular}


El promedio de las temperaturas mínimas diarias de verano es de $1,05^{\circ} \mathrm{C}$, que en este estudio se asume como el aporte del cambio climático a la estimación del ICU futura mediante el modelo de Oke (Figura 2). La ciudad que más aumenta su temperatura mínima es Hanga Roa con $1,77^{\circ} \mathrm{C}$, mientras que la que menos aumenta es Valparaíso con $0,69^{\circ} \mathrm{C}$.

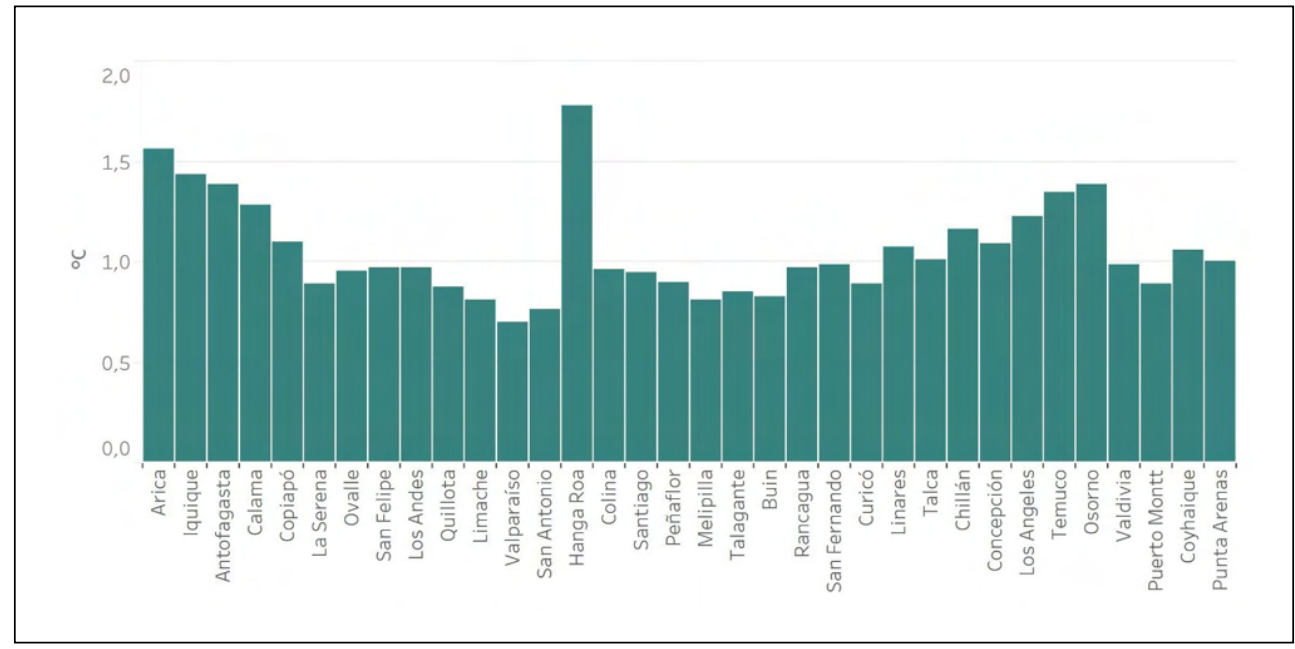

Figura 2. Variación de la temperatura mínima diaria promedio de verano (DEF) futura y presente. Ciudades ordenadas según latitud de norte (izquierda) a sur (derecha).

Fuente: datos calculados a partir de base de Meteodata.

En Santiago se proyecta la ICU más intensa $\left(10,4^{\circ} \mathrm{C}\right)$ para el año 2065 , luego le siguen Gran Concepción, La Serena - Coquimbo y Antofagasta con valores en torno a los $5,3^{\circ} \mathrm{C}$. Las ICU más bajas corresponderían a Hanga Roa, Punta Arenas y Coyhaique con valores entre 2,6 y $2,9^{\circ} \mathrm{C}$, respectivamente (Tabla 2 ).

En todas las ciudades se proyecta un aumento de la ICU, destacando las ciudades de San Felipe, Hanga Roa y Colina con un aumento esperado por sobre $1,9^{\circ} \mathrm{C}$. Por el contrario, Valparaíso, Peñaflor y San Antonio muestran el menor crecimiento de la intensidad de la ICU con valores bajo $1,0{ }^{\circ} \mathrm{C}$. La distribución espacial muestra que Santiago, capital del país, tiene la mayor magnitud de intensidad de ICU (Figuras 3 y 4 ).

De acuerdo con una clasificación de intensidad de ICU realizada a partir de los resultados obtenidos para las ciudades chilenas para ambos períodos, es posible observar que el número de casos de ICU fuerte aumenta de 1 en el presente a 14 en el futuro y la ICU moderada de 14 a 19 casos. Mientras que la ICU débil disminuye de 19 a sólo 1 caso, siendo la ciudad de Hanga Roa la única que se mantiene en esta categoría (Tabla 3). 


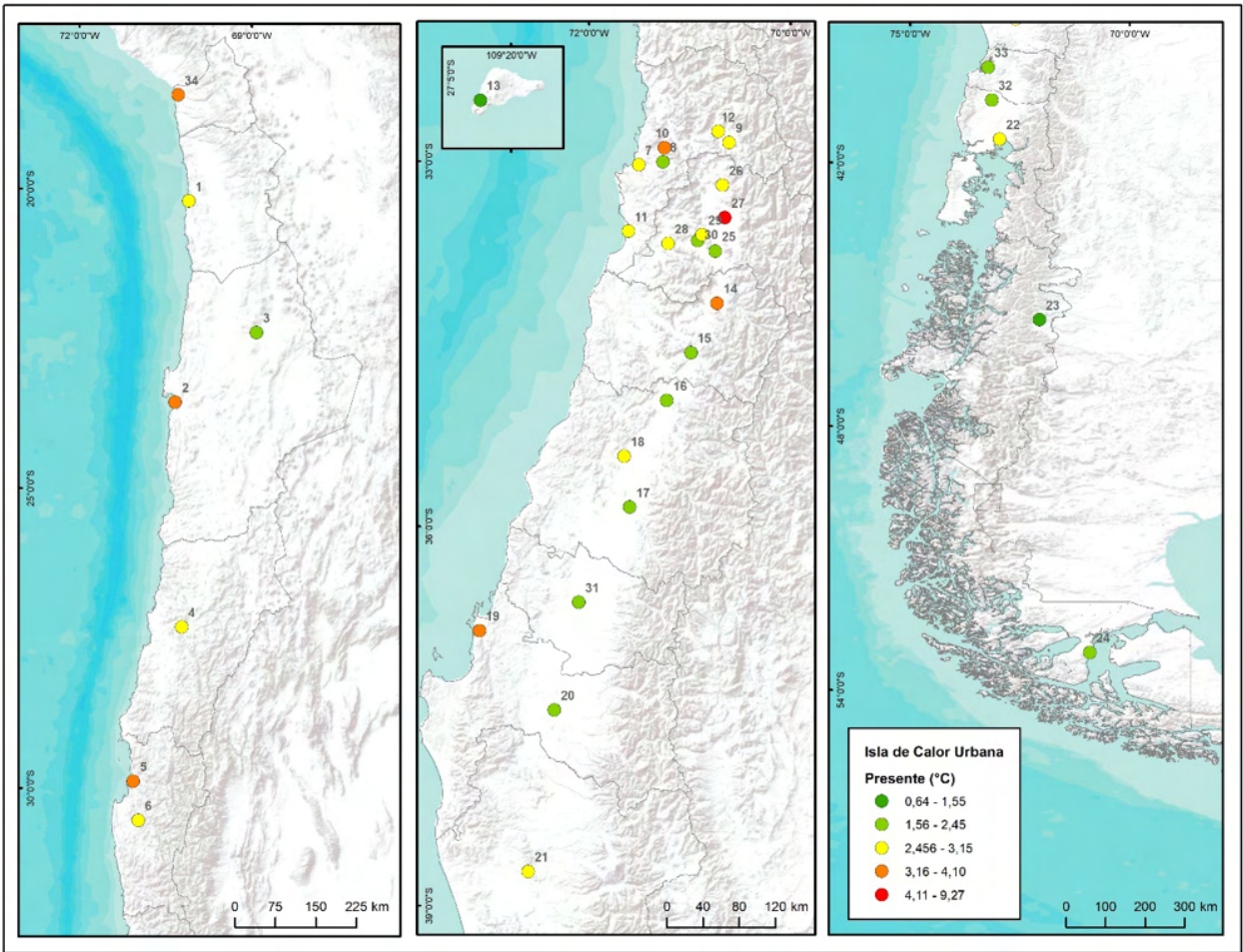

Figura 3. Isla de Calor Urbana presente.

Fuente: Elaboración propia.

Nota. Los números en el mapa indican la ciudad de acuerdo con el № señalado en la Figura 1.

Tabla 3. Clasificación de la intensidad de ICU según número de casos presente y futuro

\begin{tabular}{|l|c|c|c|}
\hline \multicolumn{2}{|c|}{ Temperatura } & Presente & Futuro \\
\hline Intensidad & Intervalo $\left({ }^{\circ} \mathrm{C}\right)$ & $N^{o}$ casos & $N^{o}$ casos \\
\hline Débil & $0,64-2,59$ & 19 & 1 \\
Moderada & $2,6-4,10$ & 14 & 196 \\
Fuerte & $4,11-10,49$ & 1 & 14 \\
\hline
\end{tabular}

Fuente: Elaboración propia. 


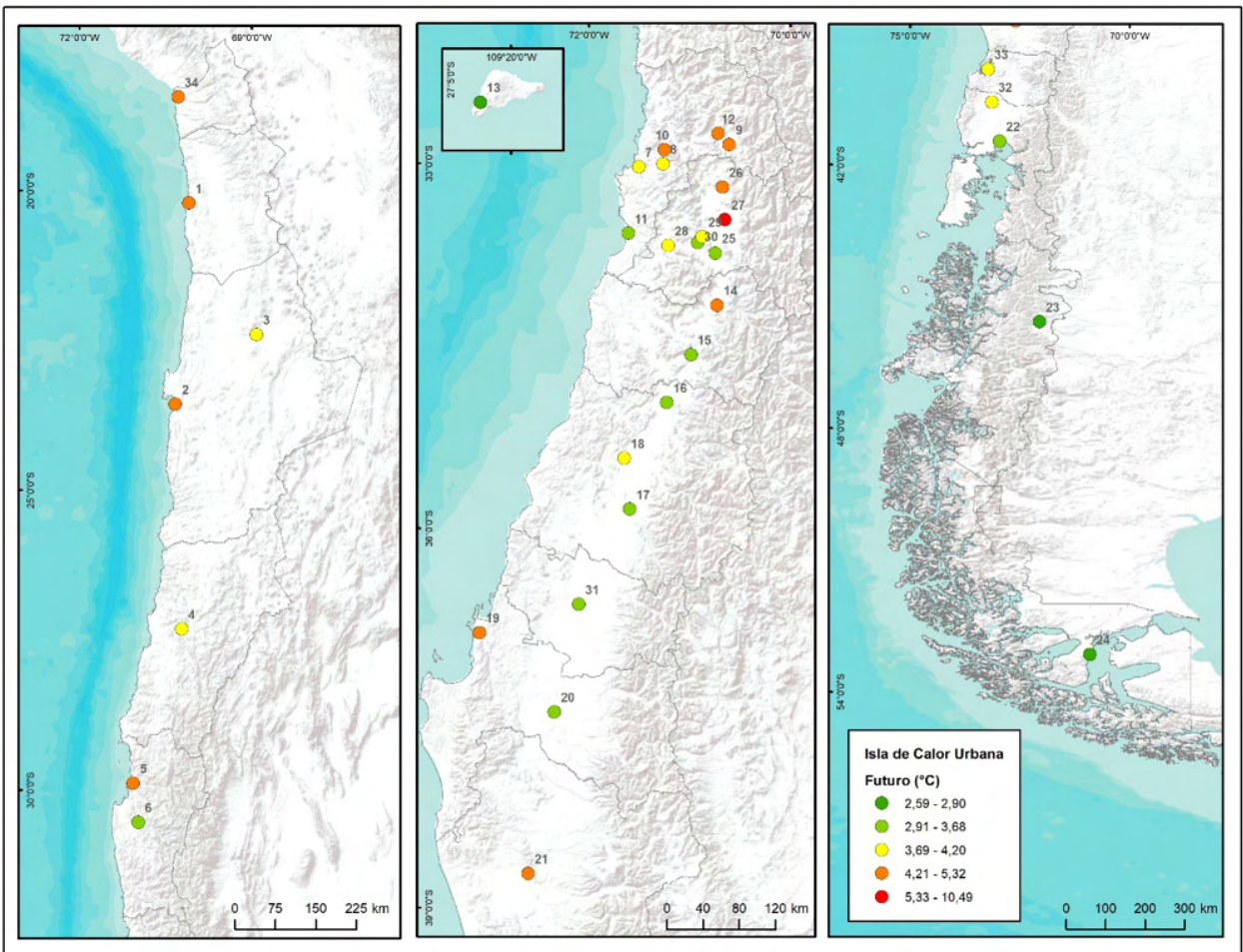

Figura 4. Isla de Calor Urbana futura.

Fuente: Elaboración propia.

Nota. Los números en el mapa indican la ciudad de acuerdo con el № señalado en la Figura 1.

\subsection{Validación de la ICU}

Para la validación de los datos de la ICU se usaron distintas aproximaciones como se indicó en la metodología. En primer lugar, se usaron los datos de temperatura mínima del aire $\left({ }^{\circ} \mathrm{C}\right)$ de las celdas rurales y urbanas definidas por Meteodata como proxy de la ICU para el período presente. En este sentido la tabla 4 compara los datos de ICU obtenidos con la fórmula de Oke con aquella hallada con la temperatura de Meteodata y la isla de calor superficial (ICUs) obtenida con la temperatura nocturna de imágenes satelitales

De acuerdo a los resultados de Meteodata, solo la ciudad de Santiago supera $1{ }^{\circ} \mathrm{C}$, alcanzando una intensidad de $2,4^{\circ} \mathrm{C}$, que a su vez posee la mayor diferencia con la intensidad de la ICU obtenida a partir de la ecuación de Oke. La menor diferencia se observa en la ciudad de Antofagasta, que además es la única que posee menos de un grado de diferencia $\left(0,84^{\circ} \mathrm{C}\right)$. La diferencia promedio entre la ICU calculada con Me- 
teodata y la ICU obtenida con la fórmula de Oke alcanza $2,5^{\circ} \mathrm{C}$. Con los datos de Meteodata, en la mayoría de las ciudades el valor de la ICU aumenta en el futuro, sin embargo, el aumento es inferior a un punto decimal, y por lo tanto no es representativo de este fenómeno urbano-climático.

Una de las causas que puede explicar este bajo ajuste de los datos de Meteodata se debe al tamaño del píxel utilizado que corresponde a 5 x $5 \mathrm{~km}$, derivado de un proceso de downscaling de modelos climáticos globales de menor resolución. En la mayoría de las ciudades estudiadas, los píxeles que contienen los puntos rurales y urbanos abarcan tanto superficie urbana como rural, influyendo sobre los datos de temperatura. En efecto, se observa que algunas ciudades registran una ICU negativa y valores muy cercanos a 0 , lo que puede deberse a la baja resolución de las celdas de la base de Meteodata que no permite distinguir claramente las coberturas urbanas y rurales. Además, es importante mencionar que los datos de Meteodata no son horarios, por lo que se descarta como método de validación de la ICU. Esto demuestra que la resolución espacial y temporal de los datos de Meteodata no son apropiados para estimar la ICU.

Esta dificultad se podría subsanar usando una aproximación metodológica distinta, como lo son las imágenes satelitales térmicas nocturnas (temperatura superficial del suelo). Se encuentran similitudes, sobre todo al comparar la ICUs nocturna con los valores obtenidos con la fórmula de Oke para el presente, en este caso la diferencia promedio hallada es de $1,0^{\circ} \mathrm{C}$ (Tabla 4).

El ajuste con la ICU de Oke aumenta si se observan los resultados de la ICU nocturna promedio de verano calculada para 10 ciudades escogidas, como se observa en la Tabla 5. En este caso, se agregan, además de los datos de estaciones meteorológicas oficiales, los datos de la ICU diurna y nocturna calculadas con registradores HOBO, instalados en algunas ciudades chilenas, que miden la temperatura del aire cada una hora. Con estos datos la diferencia se reduce significativamente, generando por lo mismo un mayor ajuste con los resultados encontrados a partir de la fórmula de Oke. Por ejemplo, en el caso de Santiago el ajuste de la ICU con datos de registradores HOBO es bastante cercano $\left(6,8^{\circ} \mathrm{C}\right)$ al estimado con la fórmula de Oke $\left(9,27^{\circ} \mathrm{C}\right)$.

En una escala espacial más detallada, se puede observar la gran variabilidad interna de la ICU promedio y máxima considerando la localización de cada instrumento en la ciudad de Santiago (Figura 5). Los valores asociados a cada punto se encuentran además en las tablas que acompañan el mapa, dando cuenta también de la variación horaria de la ICU en un mismo punto, reafirmando que la mayor intensidad se encuentra en la noche. Si bien es cierto, los valores promedio de la ICU varían entre 0,5 y $5-6^{\circ} \mathrm{C}$, dependiendo de la ciudad y el punto dentro de esta, cuando se analizan los valores máximos, encontramos diferencias urbano-rurales entre 5 y $10^{\circ} \mathrm{C}$ en incluso superiores a $\operatorname{los} 10^{\circ} \mathrm{C}$, como se observa en el Gran Santiago. 
Tabla 4. Validación de ICU $\left({ }^{\circ} \mathrm{C}\right)$

\begin{tabular}{|c|c|c|c|c|c|}
\hline \multirow[b]{2}{*}{ Ciudades } & \multirow{2}{*}{$\begin{array}{c}\text { ICU Oke } \\
\text { Presente }\left({ }^{\circ} \mathrm{C}\right)\end{array}$} & \multirow{2}{*}{$\begin{array}{l}\text { ICU Meteodata }{ }^{*} \\
\text { Presente }\left({ }^{\circ} \mathrm{C}\right)\end{array}$} & \multirow{2}{*}{$\begin{array}{l}\text { ICUs Satelital** } \\
\text { Presente }\left({ }^{\circ} \mathrm{C}\right)\end{array}$} & \multicolumn{2}{|c|}{ Diferencias ICU $\left({ }^{\circ} \mathrm{C}\right)$} \\
\hline & & & & $\begin{array}{c}\text { Oke-- } \\
\text { Meteodata }\end{array}$ & $\begin{array}{c}\text { Oke-ICU } \\
\text { Satelital }\end{array}$ \\
\hline Arica & 3,43 & $-0,26$ & 1,5 & 3,69 & 1,93 \\
\hline Iquique & 3,14 & 0,70 & 2,2 & 2,44 & 0,94 \\
\hline Antofagasta & 3,64 & 0,57 & 2,8 & 0,84 & 0,84 \\
\hline Calama & 2,41 & 0,08 & 3,1 & 2,33 & $-0,69$ \\
\hline Copiapó & 2,56 & 0,37 & $-1,1$ & 2,19 & 3,66 \\
\hline La Serena & 3,88 & 0,46 & 1,7 & 3,42 & 2,18 \\
\hline Ovalle & 2,54 & 0,47 & 1,7 & 2,07 & 1,44 \\
\hline San Felipe & 2,99 & $-0,17$ & 3,2 & 3,16 & $-0,21$ \\
\hline Los Andes & 3,04 & 0,26 & 2,6 & 2,78 & 0,44 \\
\hline Quillota & 3,64 & $-0,09$ & 2,2 & 3,73 & 1,44 \\
\hline Limache & 2,32 & 0,64 & 1,8 & 1,68 & 0,52 \\
\hline Gran Valparaíso & 3,12 & 0,45 & 1,8 & 2,67 & 1,32 \\
\hline San Antonio & 2,54 & 0,78 & 2,7 & 1,76 & $-0,16$ \\
\hline Colina & 2,92 & 0,20 & 1,7 & 2,72 & 1,22 \\
\hline Gran Santiago & 9,27 & 2,41 & 4,1 & 6,86 & 5,17 \\
\hline Peñaflor & 2,85 & $-0,33$ & 0,9 & 3,18 & 1,95 \\
\hline Melipilla & 2,55 & 0,29 & 1,4 & 2,26 & 1,15 \\
\hline Talagante & 2,29 & $-0,02$ & 1,8 & 2,31 & 0,49 \\
\hline Buin & 2,23 & 0,76 & 2,2 & 1,47 & 0,03 \\
\hline Rancagua & 3,66 & 0,28 & 2,5 & 3,38 & 1,16 \\
\hline San Fernando & 2,24 & 0,35 & 1,5 & 1,89 & 0,74 \\
\hline Curicó & 2,39 & 0,17 & 2,2 & 2,22 & 0,19 \\
\hline Linares & 2,00 & 0,33 & 1,6 & 1,67 & 0,4 \\
\hline Talca & 2,80 & 0,68 & 2,4 & 2,12 & 0,4 \\
\hline Chillán & 2,21 & 0,40 & 1,4 & 1,81 & 0,81 \\
\hline Gran Concepción & 4,10 & 0,79 & 2,1 & 3,31 & 2,0 \\
\hline Los Ángeles & 2,01 & 0,19 & 1,2 & 1,82 & 0,81 \\
\hline Temuco & 3,15 & 0,11 & 2,3 & 3,04 & 0,85 \\
\hline Osorno & 2,30 & 0,24 & 2,8 & 2,06 & $-0,5$ \\
\hline Valdivia & 2,45 & 0,06 & 2,5 & 2,39 & $-0,05$ \\
\hline Puerto Montt & 2,54 & 0,22 & 0,6 & 2,32 & 2,48 \\
\hline Coyhaique & 1,55 & $-0,09$ & 1,7 & 1,64 & $-0,15$ \\
\hline Punta Arenas & 1,81 & 0,25 & 1,2 & 1,56 & 0,61 \\
\hline
\end{tabular}

Fuente: Elaboración propia en base a información obtenida de Meteodata, instrumental (estaciones meteorológicas SINCA, DMC y AGROMET) y resultados Fondecyt iniciación 11180990.

Nota: * ICU Meteodata corresponde al cálculo de la ICU a partir de los datos de temperatura mínima promedio de verano proporcionadas por Meteodata.

** ICU superficial corresponde a la Isla de Calor Urbana Superficial calculada a partir del promedio de temperatura superficial nocturna promedio 2008-2018, obtenido sobre imágenes satelitales MODIS.

Las columnas en gris corresponden a la ICU presente calculada en este estudio a partir de la fórmula de Oke (1987). 
Tabla 5. Valores según ICU instrumental, registros $\mathrm{HOBO}$ y estimación Oke $\left({ }^{\circ} \mathrm{C}\right)$

\begin{tabular}{|c|c|c|c|c|c|c|c|}
\hline \multirow[b]{2}{*}{ Ciudades } & \multirow{2}{*}{$\begin{array}{c}\text { ICU Oke } \\
\text { Presente } \\
\left({ }^{\circ} \mathrm{C}\right)\end{array}$} & \multicolumn{2}{|c|}{ ICU instrumental } & \multicolumn{2}{|c|}{ ICU HOBO*** } & \multicolumn{2}{|c|}{ Diferencia ICU $\left({ }^{\circ} \mathrm{C}\right)$} \\
\hline & & $\begin{array}{c}\text { Diurna } \\
\left({ }^{\circ} \mathrm{C}\right)\end{array}$ & $\begin{array}{c}\text { Nocturna } \\
\left({ }^{\circ} \mathrm{C}\right)\end{array}$ & $\begin{array}{c}\text { Diurna } \\
\left({ }^{\circ} \mathrm{C}\right)\end{array}$ & $\begin{array}{c}\text { Nocturna } \\
\left({ }^{\circ} \mathrm{C}\right)\end{array}$ & $\begin{array}{c}\text { Oke- } \\
\text { Instrumental } \\
\text { (nocturna) }\end{array}$ & $\begin{array}{c}\text { Oke- } \\
\text { HOBO } \\
\text { (nocturna) }\end{array}$ \\
\hline Arica & 3,43 & $-0,4$ & $-0,3$ & & & 3,73 & \\
\hline Antofagasta & 3,64 & $-0,2$ & 1,0 & 3,9 & 3,9 & 2,64 & $-0,26$ \\
\hline Calama & 2,41 & 0,5 & 1,6 & & & 0,81 & \\
\hline Los Andes & 3,04 & 1,8 & 3,6 & & & $-0,56$ & \\
\hline Gran Valparaíso & 3,12 & & & 4,6 & 3,9 & - & $-0,78$ \\
\hline Gran Santiago & 9,27 & 5,1 & 3,0 & 4,4 & 6,8 & 6,27 & 2,47 \\
\hline Curicó & 2,39 & 1,0 & 3,4 & & & $-1,01$ & \\
\hline Talca & 2,80 & 1,9 & 3,3 & & & $-0,5$ & \\
\hline Chillán & 2,21 & 1,9 & 1,4 & 4,5 & 2,5 & 0,81 & $-0,29$ \\
\hline Gran Concepción & 4,10 & & & 4,5 & 5,8 & - & $-1,7$ \\
\hline Osorno & 2,30 & $-0,5$ & 1,0 & & & 1,3 & \\
\hline Coyhaique & 1,55 & 0,9 & 0,5 & & & 1,05 & \\
\hline Punta Arenas & 1,81 & 0,0 & 0,5 & & & 1,31 & \\
\hline
\end{tabular}

Fuente: Elaboración propia.

Nota: Las columnas en gris corresponden a la ICU presente calculada en este estudio a partir de la fórmula de Oke (1987).

* ICU instrumental corresponde a aquella calculada a partir de los datos de estaciones meteorológicas fijas pertenecientes a redes de monitoreo oficiales, urbanas y rurales.

** ICU instrumental corresponde a aquella calculada a partir de los datos de temperatura obtenidos a partir de registradores HOBO.

\section{Discusión y conclusiones}

Los resultados obtenidos permiten validar el método propuesto para determinar la ICU presente y futura. Si bien, la ICU no es el impacto más extremo de cambio climático en las ciudades, es uno de los más representativos del proceso de urbanización y del clima urbano, especialmente para las metrópolis, grandes ciudades y ciudades medias, como las estudiadas.

En términos comparativos el modelo adaptado de Oke para estimar la ICU futura es acorde a los resultados obtenidos por estudios globales considerando los efectos del cambio climático (Manoli et al., 2019; Zhao et al., 2021). El estudio de Zhao et al (2021) pronostica una aumento de $4 \stackrel{\circ}{\circ}$ para las áreas urbanas a nivel global, lo que es consistente con los 4,6 ${ }^{\circ} \mathrm{C}$ para el conjunto de ciudades chilenas evaluadas. 

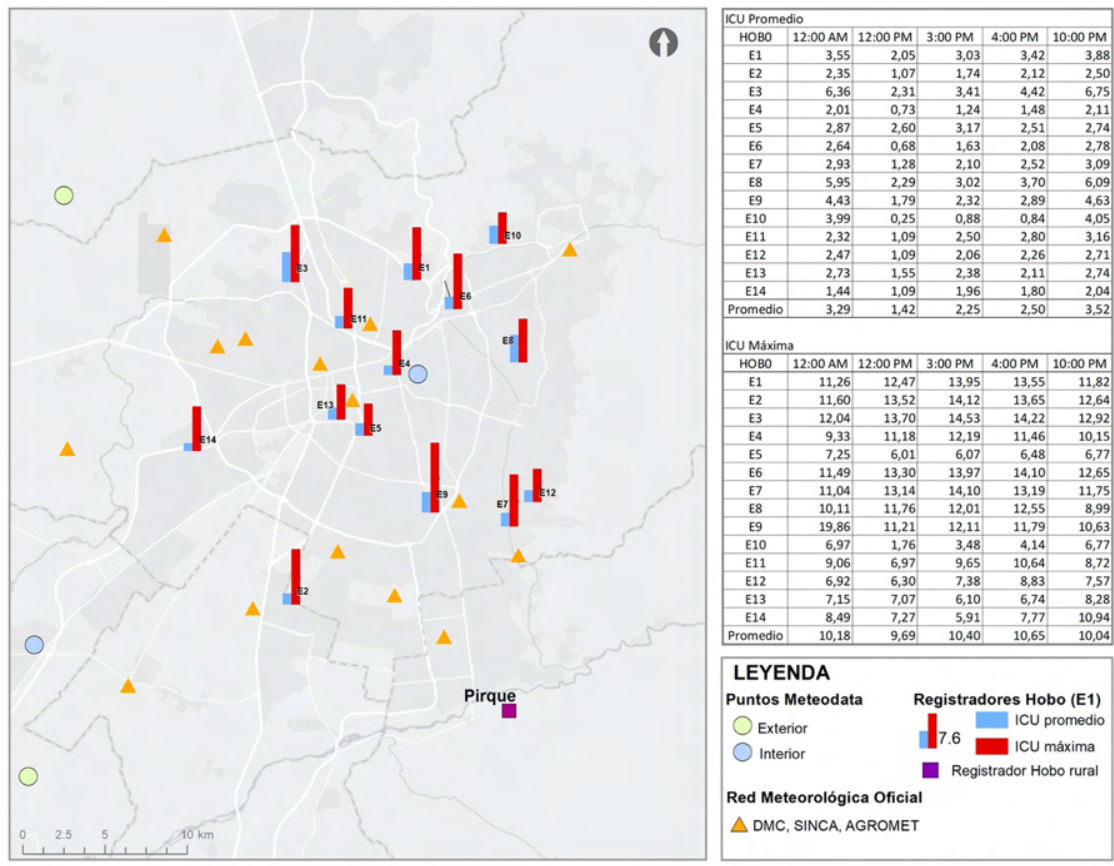

Figura 5. Valores de ICU actuales (período 2013-2018) en el Gran Santiago $\left({ }^{\circ} \mathrm{C}\right)$. La ICU promedio nocturna y la ICU máxima están calculadas para la estación de verano (DEF).

Fuente: Elaboración propia.

Se sabe que las ciudades ejercen una influencia significativa en su clima local, ya que generalmente son más cálidas que su entorno. Sin embargo, los modelos climáticos generalmente no incluyen una representación adecuada de las áreas urbanas, por lo que es probable que las proyecciones climáticas de los modelos subestimen las temperaturas en las zonas urbanas (Kershaw et al., 2010). En efecto, en este caso los datos de Meteodata se basan en modelos climáticos globales que usan celdas de baja resolución (Meteodata, 2020). De esta forma, a pesar del proceso de downscaling, no es posible determinar con precisión las propiedades urbanas y rurales, especialmente para ciudades medias y pequeñas, y por tanto no se puede estimar la intensidad de la ICU de manera directa.

En los últimos años ha aumentado el número de investigaciones que intentan modelar la ICU futura. Estudios desarrollados a partir de los modelos regionales de simulación, como el EURO-CORDEX, para Europa (Atenas, Grecia, en Van der Schriek et al, 2020; Lisboa, Portugal en Nogueira et al, 2020), reconocen la necesidad de realizar un downscaling que considere factores locales. Algunas investigaciones complementan los modelos de simulación con modelos que representan las características urbanas, como por ejemplo el estudio de Lauwaet et al (2016), quienes incorporan un modelado del dosel urbano a muy ata resolución. 
Si bien la ICU es en general más intensa durante la noche, un importante número de investigaciones además de proyectar una ICU nocturna, considerando valores de temperatura mínima, también estiman la ICU diurna, considerando valores de temperatura máxima (Nogueira et al. 2020; Van der Schriek et al, 2020).

Al igual que nuestro estudio, el estudio conducido por Huang et al (2019) para un conjunto de ciudades ubicadas en diferentes dominios climáticos se basó en el crecimiento urbano proyectado para el año 2050. Los resultados del estudio prevén un crecimiento de entre 0,6 y 1,3 millones de $\mathrm{km}^{2}$ de áreas urbanas entre 2015 y 2050, resultando en un calentamiento medio durante el día y la noche en verano con una temperatura del aire de $0,5^{\circ} \mathrm{C}$ a $0,7^{\circ} \mathrm{C}$, hasta $\pm 3{ }^{\circ} \mathrm{C}$. Este aumentará los riesgos de calor extremo para aproximadamente la mitad de la población urbana futura, principalmente en el sur global tropical, donde los pronósticos existentes ya indican un calentamiento de emisiones de gases de efecto invernadero más fuerte y una falta de capacidad de adaptación.

El modelo acoplado de proyección meteorológica y dosel urbano aplicado en el área metropolitana de Beijing-Tianjin-Hebei, desde la década de 1980 hasta 2005 y proyectado al futuro para el año 2050, señala que, debido a la expansión urbana, como mínimo la ICU (nocturna) aumentaría alrededor de $5 \stackrel{\circ}{\circ}$ en las áreas recientemente desarrolladas, Mientras que en las áreas urbanas más antiguas y consolidadas aumentaría un grado más (Wang et al 2016). Por el contrario, en la ciudad de Bruselas, los resultados mostraron una leve disminución de la ICU, lo que se asocia al aumento de la temperatura del entorno rural de la ciudad de Bruselas (Lauwaet et al 2016).

En Lisboa la ICU media anual actual fue $\pm 3^{\circ} \mathrm{C}$, y el modelo para el periodo 20212050 muestra un calentamiento de $\pm 1^{\circ} \mathrm{C}$ para y $\pm 3,5^{\circ} \mathrm{C}$ para $2070-2099$. Además, la ICU de Lisboa exhibe un efecto de estacionalidad: durante la noche la ICU fue máxima durante el invierno $\left( \pm 4^{\circ} \mathrm{C}\right)$ y mínimo en verano $\left.\pm 2^{\circ} \mathrm{C}\right)$; durante el día, la ICU cambió la señal entre $\pm 1^{\circ} \mathrm{C}$ durante el invierno y $\pm-1{ }^{\circ} \mathrm{C}$ durante el verano (Nogueira et al. 2020). En Grecia calculando la intensidad de la ICU mediante temperaturas mínimas nocturnas se demuestra que es mucho más intensa que usando las temperaturas máximas diurnas (Van der Schriek et al, 2020). Del mismo modo en Delhi, se aplica un modelo climático urbano para realizar proyecciones climáticas a corto y largo plazo, considerando el escenario RCP8.5. La intensidad de la ICU, basada en el 5\% de las noches más cálidas (TNp95), fue de $4,1^{\circ} \mathrm{C}$ y presenta un cambio insignificante a lo largo del tiempo. Sin embargo, los resultados indican claramente la contribución de los efectos de la ICU en la intensificación de los impactos del calor extremo y el estrés térmico en la ciudad.

En el caso de Santiago de Chile, el estudio de Sarricolea Martín-Vide (2014), evaluando la Isla de Calor Urbana de Superficie (ICUs) mediante Análisis de Componentes Principales de temperaturas de emisión para 53 imágenes Terra MODIS, identifica intensidades superiores a $5^{\circ} \mathrm{C}$, localizándose los valores máximos en las comunas centrales y de Huechuraba y Quilicura. 
Por otro lado, un resultado de gran interés corresponde al modelo de cambio de uso de suelo urbano aplicado en esta investigación. Este pronostica un aumento de 98.565 nuevas hectáreas urbanas que equivaldría a 3.301.079 nuevos habitantes urbanos al 2065 en las 34 ciudades analizadas, lo cual representa un aumento de un $27 \%$ de la exposición frente a las amenazas del cambio climático. Además, el modelo plantea un crecimiento sostenido de las manchas urbanas y consolidación de conglomerados urbanos, pasando de $180.641,2$ ha en la actualidad a $278.246,6$ ha al año 2065 , es decir, un crecimiento bruto de 97.605, 4 ha urbanas. Como limitante del modelo de estimación de población aplicado para aplicar la fórmula de Oke, se puede mencionar que hay ciertas localidades sobreestimadas en su dato de población. Por ejemplo, la ciudad de Placilla aparece en segundo lugar, luego de Santiago, con una población cercana a los 2 millones de habitantes, debido al gran crecimiento de población entre los años 1992 y 2017, lo que resulta ser un valor sobreestimado. Un caso similar ocurre con la localidad de Ventanas que pasaría de 5.029 habitantes a sobre 375.000 habitantes al 2065, ya que el modelo de simulación proyecta una superficie casi 10 veces mayor a la registrada al año 2002.

En relación con el impacto por aumento de intensidad de ICU se puede afirmar que Santiago, por su gran extensión, es la ciudad con mayor impacto tanto presente como futuro. La validación con datos de otras fuentes reafirma tal tendencia. Este fenómeno también se repite en grandes ciudades y ciudades de porte medio, lo que plantea grandes desafíos para su mitigación a través de medidas que no sean perjudiciales para el cambio climático, como son, el uso de combustible fósil para usar aire acondicionado. Sin duda las viviendas más precarias como los campamentos serán los más afectados por la ICU, y dentro de estas la población más vulnerable y expuesta como son los adultos mayores y niños. Se puede concluir que las ciudades de valles interiores serán las más afectadas por la ICU, especialmente de la zona central del país.

De acuerdo al estudio Romero (2019), existe una clara relación entre relación entre la distribución espacial de las temperaturas urbanas, el material particulado y los grupos socioeconómicos. En el caso de Santiago los pobres viven en lugares con ICU más intensas, donde la falta de vegetación seca el aire y, por lo general, ocupan zonas con menos ventilación, lo que, en consecuencia, provoca una mayor concentración de contaminantes atmosféricos. En cambio, los habitantes más ricos están acostumbrados a vivir en zonas de islas urbanas más frías donde las temperaturas no son tan elevadas debido a la presencia de jardines, bosques urbanos y parques. En este sentido, la consideración del clima urbano debe formar parte de los procesos de planificación territorial para lograr una mejor "calidad del clima" en los asentamientos urbanos (Alcofarado, y Matzarakis, 2014), lo cual es un gran desafío para los países latinoamericanos.

Finalmente se puede destacar que esta propuesta metodológica para estimar la ICU es recomendable de usar en los diferentes instrumentos de planificación urbana y ambiental, de modo de dimensionar los impactos del cambio climático en espacios urbanos y de esta forma proponer medidas de adaptación adecuadas, especialmente en las ciudades de mayor crecimiento. 


\section{Agradecimientos}

Este trabajo se enmarca dentro del proyecto de Atlas de Riesgos Climáticos (ARClim) del Ministerio de Medio Ambiente de Chile. Se agradece especialmente a René Garreaud, Francisco Meza, Mark Falvey y Andrés Pica, así como al proyecto Fondecyt/ANID № 1180268 "Proyección de escenarios de crecimiento en ciudades medias. Modelos de simulación espacial para la planificación urbana-ambiental" (ANID) y al proyecto Fondecyt de Iniciación/ANID No 11180990 "La construcción del clima urbano: hacia la calidad y justicia climática en las ciudades chilenas". Este trabajo reconoce también el apoyo proporcionado por el CEDEUS, ANID/ FONDAP 15110020. Finalmente queremos agradecer enormemente a los pares revisores que han permitido mejorar sustantivamente el trabajo presentado.

\section{Bibliografía}

Alcofarado, M.J. \& Matzarakis, A. (2014). Planning with urban climate in different climatic zones. Geographicalia, (57). https://doi.org/10.26754/ojs_geoph/geoph.201057808

Henríquez, C., Quense, J., Contreras, P., Guerrero, N., Smith, P. \& González, F. (2020). Informe Proyecto ARClim: Ciudades. Santiago: Centro de Cambio Global UC, Centro de Ciencia del Clima y la Resiliencia y CEDEUS coordinado por Centro de Ciencia del Clima y la Resiliencia y Centro de Cambio Global UC para el Ministerio del Medio Ambiente a través de la Deutsche Gesellschaft fur Inte.

Henríquez, C., Qüense, J., Villarroel, C. \& Mallea, C. (2019). 50-years of climate extreme indices trends and inventory of natural disasters in Chilean cities (1965-2015). In Cristián Henríquez \& H. Romero (Eds.), Urban Climate in Latin America (pp. 281-308). Cham: Springer Nature. https://doi.org/https://doi.org/10.1007/978-3-319-97013-4_6

Henríquez, C. (2014). Modelando el crecimiento de ciudades medias. Hacia un desarrollo urbano sustentable. Santiago: Ediciones UC, Colección Textos Universitarios.

Huang, K., Li, X., Liu, X. \& Seto, K.C. (2019). Projecting global urban land expansion and heat island intensification through 2050. Environmental Research Letters, 14(11). https://doi.org/10. 1088/1748-9326/ab4b71

INE (2019). Ciudades, Pueblos, Aldeas y Caseríos 2019. Santiago de Chile.

INE \& MINVU (2018). Metodología para medir el crecimiento urbano de las ciudades de Chile. Santiago de Chile: Comisión de Estudios Hitacionales y Urbanos del Ministerio de Vivienda y Urbanismo.

Kershaw, T., Sanderson, M., Coley, D. \& Eames, M. (2010). Estimation of the urban heat island for UK climate change projections. Building Services Engineering Research \& Technology, 31(3), pp. 251-263. https://doi.org/Doi 10.1177/0143624410365033

Lauwaet, D., De Ridder, K., Saeed, S., Brisson, E., Chatterjee, F., van Lipzig, N.P.M., ... Hooyberghs, H. (2016). Assessing the current and future urban heat island of Brussels. Urban Climate, 15. https://doi.org/10.1016/j.uclim.2015.11.008 
Manoli, G., Fatichi, S., Schläpfer, M., Yu, K., Crowther, T. W., Meili, N., ... Bou-Zeid, E. (2019). Magnitude of urban heat islands largely explained by climate and population. Nature. https:// doi.org/10.1038/s41586-019-1512-9

Nogueira, M., Lima, D. \& Soares, P. (2020). An integrated approach to project the future urban climate response: Changes to Lisbon's urban heat island and temperature extremes. Urban Climate, 34. https://doi.org/10.1016/j.uclim.2020.100683.

Ochoa de la Torre, J.M.; Marincic Lovriha. I.; Urcelay Sánchez, M.A. (2009). Índices de confort térmicos en la planeación de sitios turísticos. Topofilia: Revista de Arquitectura, Urbanismo y Ciencias Sociales Centro de Estudios de América Del Norte, El Colegio de Sonora, I(3), pp. 1-12.

Oke, T.R. (1987). Boundary Layer Climates (2.nd ed.). London: Routledge Print. https://doi.org/ 10.1016/0012-8252(90)90005-G

Romero (2019). Urban Climates and the Challenge of Sustainable Development of Chilean Cities. En: Henríquez, C. \& Romero, H. (Ed.) Urban Climates in Latin America. Springer.Nature, Cham, Switzerland: pp. 111-134. ISBN 978-3-319-97012-7, https://doi.org/10.1007/978-3-31997013-4_9

Romero, H., Ordenes, F. \& Vásquez, A. (2003). Ordenamiento territorial y desarrollo sustentable a escala regional, ciudad de Santiago y ciudades intermedias en Chile. In E. Figueroa \& J. Simonetti (Eds.), Globalización y Biodiversidad: Oportunidades y desafios para la sociedad chilena (pp. 167-224). Santiago: Programa Interdisciplinario de Estudios en Biodiversidad (PIEB), Universidad de Chile.

Sarricolea, P., Herrera-Ossandon, M. \& Meseguer-Ruiz, Ó. (2017). Climatic regionalisation of continental Chile. Journal of Maps, 13(2), pp. 66-73. https://doi.org/10.1080/17445647.2016.1259592

Sarricolea, P. \& Martín-Vide, J. (2014). El estudio de la Isla de Calor Urbana de Superficie del Área Metropolitana de Santiago de Chile con imágenes Terra-MODIS y Análisis de Componentes Principales. Revista de Geografia Norte Grande, (57). https://doi.org/10.4067/s0718-34022014 000100009

Smith, P. \& Romero, H. (2016). Factores explicativos de la distribución espacial de la temperatura del aire de verano en Santiago de Chile. Revista de Geografia Norte Grande. https:// doi.org/10.4067/s0718-34022016000100004

Van der Schriek, T., Varotsos, K., Giannakopoulos, C. \& Founda, F. (2020). Projected Future Temporal Trends of Two Diferent Urban Heat Islands in Athens (Greece) under Three Climate Change Scenarios: A Statistical Approach. Atmosphere, 11, 637. Doi: 10.3390/atmos11060637

Wang, J., Huang, B., Fu, D., Atkinson, P.M. \& Zhang, X. (2016). Response of urban heat island to future urban expansion over the Beijing-Tianjin-Hebei metropolitan area. Applied Geography, 70. https://doi.org/10.1016/j.apgeog.2016.02.010

Whitford, V., Ennos, A.R. \& Handley, J.F. (2001). "City form and natural process" - indicators for the ecological performance of urban areas and their application to Meryside, UK. Landscape and Urban Planning, 57, pp. 91-103.

Zhao, L., Oleson, K., Bou-Zeid, E., Krayenhoff, E.S., Bray, A., Zhu, Q., ... Oppenheimer, M. (2021). Global multi-model projections of local urban climates. Nature, s41558-020-00958-8. https://doi.org/https://doi.org/10.1038/s41558-020-00958-8 